\title{
Espécies de flebotomíneos (Diptera, Psychodidae) coletadas em ambiente urbano em municípios com transmissão de Leishmaniose Visceral do Estado de Mato Grosso do Sul, Brasil
}

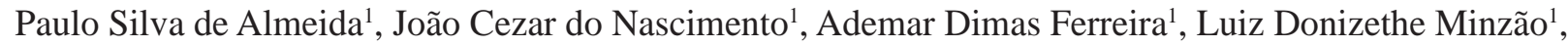 \\ Francisco Portes ${ }^{1}$, Airton Marques de Miranda ${ }^{1}$, Odival Faccenda ${ }^{2}$ \& José Dilermando Andrade Filho ${ }^{3}$
}

${ }^{1}$ Laboratório Regional de Entomologia, Núcleo Regional de Saúde da Secretaria Estadual de Saúde, Rua Hilda Bergo Duarte, 940, Centro, 79806-020 Dourados-MS, Brasil.

${ }^{2}$ Universidade Estadual de Dourados, Rod. Dourados Itaum, km 12, Cidade Universitária, 79804-970 Dourados-MS, Brasil.

${ }^{3}$ Centro de Pesquisas René Rachou/Fiocruz-MG. Av. Augusto de Lima, 1715, Barro Preto, 30190-002 Belo Horizonte-MG, Brasil. jandrade@cpqrr.fiocruz.br

\begin{abstract}
Species of phlebotomines (Diptera, Psychodidae) collected in urban municipalities with transmission of visceral leishmaniasis in Mato Grosso do Sul State, Brazil. The phlebotomine sand flies are the natural vectors of some etiological agents of human and animal diseases, such as the protozoa of the genus Leishmania Ross, 1903. The phlebotomine fauna in Mato Grosso do Sul is relatively well known and so far consists of 54 species. The present study is based on the survey of the phlebotomine fauna of the urban area of the 18 municipal districts with transmission of visceral leishmaniasis, in the state of Mato Grosso do Sul, for the purpose of verifying the main species and providing data for the leishmaniasis control programmes. The collections were undertaken with automatic light traps, installed monthly on three consecutive nights, from 06:00 pm to 6:00 am over a two-year period. A total of 34,799 sand fly specimens, belonging to 36 species, were collected. Lutzomyia longipalpis (Lutz \& Neiva, 1912) and Nyssomyia whitmani (Antunes, 1939) were the most widely dispersed species, the former was found in 16 and the latter in 15 out of the 18 municipalities investigated, however, Lu. longipalpis was predominant in all the municipalities and Ny. whitmani was not predominant in any of them. Corumbá contributed with $40.92 \%$ of the specimens and Lutzomyia cruzi (Mangabeira, 1938) with $92.50 \%$ of the specimens captured there. It is suggested that the species of Lutzomyia genus and Ny. whitmani may be involved in the transmission of leishmaniasis in Mato Grosso do Sul state.
\end{abstract}

KEYWORDS. Cutaneous leishmaniasis; visceral leishmaniasis; Phlebotominae.

RESUMO. Os flebotomíneos são os vetores naturais de alguns agentes etiológicos de doenças humanas e de animais, tais como protozoários do gênero Leishmania Ross, 1903. A fauna flebotomínica no Mato Grosso do Sul é relativamente bem conhecida e até o momento compõe-se de 54 espécies. O presente estudo baseia-se no levantamento de flebotomíneos em área urbana de 18 municípios com transmissão de leishmaniose visceral no Estado do Mato Grosso do Sul, com objetivo de verificar as principais espécies e fornecer subsídios para o programa de controle das leishmanioses. As coletas foram realizadas com armadilhas automáticas luminosas, instaladas mensalmente durante três noites consecutivas, das 18:00 horas às 6:00, no período de dois anos. Foram coletadas 36 espécies dentre os 34.799 exemplares identificados. Lutzomyia longipalpis (Lutz \& Neiva, 1912) e Nyssomyia whitmani (Antunes, 1939) foram as espécies mais dispersas, a primeira foi encontrada em 16 e a segunda em 15 dos 18 municípios investigados, contudo, Lu. longipalpis foi predominante em todos esses municípios $\mathrm{Ny}$. whitmani não predominou em nenhum deles. Corumbá contribuiu com $40.92 \%$ de todos flebotomíneos capturados e nesse município Lutzomyia cruzi (Mangabeira, 1938) respondeu por $92.50 \%$ dos exemplares coletados. Ressalta-se que as espécies do gênero Lutzomyia e Nyssomyia whitmani podem estar envolvidas com a transmissão de leishmanioses no Mato Grosso do Sul.

PALAVRAS-CHAVE. Leishmaniose tegumentar; leishmaniose visceral; Phlebotominae.

Os flebotomíneos são os vetores naturais de alguns agentes etiológicos causadores de doenças humanas e de animais, tais como protozoários do gênero Leishmania Ross, 1903. As leishmanioses são consideradas primariamente como zoonoses, podendo acometer o homem quando este entra em contato com o ciclo de transmissão do parasita, transformando-se em uma antropozoonose (Ministério da Saúde 2003). Esse grupo de insetos distribui-se por quase todas as regiões faunísticas do mundo, com aproximadamente 800 espécies já descritas, $60 \%$ na região Neotropical e cerca de 229 registradas para o Brasil (Galati et al. 2003a).

Segundo relatórios fornecidos pelo SINAN (Sistema de Informações de Agravos de Notificação, Serviço de Vigilância Epidemiológica da Secretaria Estadual de Saúde de Mato Grosso do Sul), em Mato Grosso do Sul, no período de 2002 a 2006, foram confirmados 1.137 casos de leishmaniose visceral (LV) com 50 óbitos, abrangendo 38 municípios. Entre estes, considerando-se a média de casos dos últimos cinco anos, seis foram classificados como de transmissão intensa, cinco moderada e 27 esporádica (Ministério da Saúde 2003) (Fig. 1). Com relação à leishmaniose tegumentar, foram registrados em igual período 1.151 casos. No sul do Estado, em uma unidade militar no município de Bela Vista, Dorval et al. (2006) constataram a infecção humana pela Leishmania amazonensis Lainson \& Shaw, 1972 e em estudos posteriores na área, comprovaram a presença do vetor Bichromomyia flaviscutellata (Mangabeira, 1942) (Dorval et al. 2007).

O objetivo do presente trabalho foi verificar as espécies de flebotomíneos que ocorrem em área urbana dos municípios com transmissão de leishmaniose visceral no Estado do Mato Grosso do Sul, Brasil, visando fornecer subsídios para o programa de controle das leishmanioses. 


\section{MATERIAL E MÉTODOS}

Área de estudo. O Estado de Mato Grosso do Sul está localizado na região Centro-Oeste, com superfície de 358,2 mil $\mathrm{Km}^{2}$. Limita-se a leste com Minas Gerais, São Paulo e Paraná e ao norte, com Mato Grosso e Goiás, também faz limites internacionais com a Bolívia e Paraguai. Sua população é de 2.033.855 habitantes (IBGE 2007), possui 78 municípios, divididos em quatro mesorregiões: Centro-Norte, Leste, Sudoeste e Pantanal. Sua capital é a cidade de Campo Grande.

Do ponto de vista geográfico, apresenta duas grandes unidades. O Planalto Sedimentar da Bacia do rio Paraná situado a leste e a planície do Pantanal sul-mato-grossense, a oeste. $\mathrm{O}$ primeiro apresenta-se com relevo de topografia suave, variando de 200 a 600 metros acima do nível do mar. O solo é predominantemente arenoso em suas porções do nordeste, leste e sul, com vegetação de cerrado e, mais fértil, de origem basáltica em sua área sudeste, nas bacias dos rios Brilhante, Dourados e Vacaria. A planície do Pantanal sul-mato-grossense, a segunda maior unidade geográfica do Estado, apresenta altitudes variando de 100 a 300 metros acima do nível do mar. O solo é argiloso e arenoso nas partes mais altas, podendo ser derivados de rochas calcárias e xistosas nas elevações isoladas, como na Serra da Bodoquena, que possui extensas jazidas de calcário, granitos e mármores e no Maciço de Urucum com reservas de manganês e minério de Ferro (Mato Grosso do Sul 2007).

O Clima, segundo classificação de Koppen, ocorrem em Mato Grosso do Sul dois tipos: o de maior abrangência na área é o AW (clima tropical úmido com estação chuvosa no verão e seca no inverno) e o CFA (clima mesotérmico úmido sem estiagem) em que a temperatura do mês mais quente é superior a $22^{\circ} \mathrm{C}$, apresentando no mês mais seco uma precipitação superior a $30 \mathrm{~mm}$, sendo sua área de ocorrência localizada ao sul do Estado. Todavia, de acordo com a classificação dos climas biológicos proposta por Bagnouls \& Gaussen (1957), ocorrem no Estado, três subregiões climáticas: a Termoxeroquimênica atenuada, de ocorrência na região da Bodoquena, na região centro-oeste do Estado e arredores da microrregião de Paranaíba, onde as temperaturas médias do mês mais frio estão entre $20^{\circ} \mathrm{C}$ e $24^{\circ} \mathrm{C}$ e as precipitações anuais chegam a $1.500 \mathrm{~mm}$. O período seco normalmente é de 3 meses; a mesoxeroquimênica modificada, que engloba as regiões sudoeste, centro-sul e nordeste do Estado, onde as temperaturas médias do mês mais frio, variam de $18^{\circ} \mathrm{C}$ a $20^{\circ} \mathrm{C}$, o período seco estende-se até cinco meses e a precipitação é regular entre 1.000 a $1.700 \mathrm{~mm}$. anuais; e a Eumesaxérica que incide na região sul do Estado, onde a curva térmica é sempre positiva, o período seco ausente, apresenta precipitação regulares durante o ano entre 1.400 a $1.700 \mathrm{~mm}$. As temperaturas médias do mês mais frio estão entre $14^{\circ} \mathrm{C}$ e $15^{\circ} \mathrm{C}$, sendo que as mínimas absolutas de inverno são baixas, de $4^{\circ} \mathrm{C}$ a $6^{\circ} \mathrm{C}$, nas invasões polares de inverno.

A vegetação reflete o contato e a interpenetração de três províncias florísticas: Amazônica, Chaquenha e da Bacia do Paraná, resultando em paisagens fitogeográficas muito diversificadas. Suas formações naturais vão desde campos limpos, completamente destituídos de árvores, a cerrados e até florestas de grande porte.

A análise da cobertura vegetal em 1985, mostrava um pequeno predomínio das formações naturais sobre as áreas antropizadas, sendo que cerca de $56 \%$ do território estadual mantinha-se com cobertura original ou apenas ligeiramente alterada. A razão deste fato, prende-se, à existência de extensas planícies pantaneiras, as quais se mantêm com aspectos primitivos, devido ao longo período de inundação, dificultando a sua ocupação. Essa situação, tende a se inverter e já, em alguns locais, o avanço incontrolável dos desmatamentos e a própria substituição de campos naturais por gramíneas exóticas está pondo em risco a estabilidade ambiental.

O Estado caracteriza-se por apresentar quatro regiões fitoecológicas distintas: savana (cerrado), denominação utilizada para as várias formações de estrutura predominantemente campestre, intercalada por pequenas plantas lenhosas até arbóreas e em geral serpenteadas por floresta-de-galeria; que se encontra praticamente em todo o Estado, destacando-se nas chapadas areno-argilosas que se estendem de sudeste a norte e nordeste; vegetação chaquenha (savana estépica) composta de elementos arbóreos xeromorfos, cactáceos e lenhosos, espinhosos, cobrindo um tapete gramíneo cespitoso, perene entremeado de ervas anuais, que se distribui nas áreas da depressão do rio Paraguai em toda sua extensão norte-sul e pelas planícies e Pantanal mato-grossense, nas superfícies por onde se distribuem os solos salinos; floresta estacional decidual que cobre os terrenos predominantemente calcários do Planalto da Bodoquena e as elevações da depressão do rio Paraguai, compreendidos entre as altitudes de 100 a 150 metro acima do nível do mar e floresta estacional semidecidual, situada na porção sul e sudeste do Estado, recobrindo os terrenos mais elevados e de litologias mais antigas.

As áreas das formações pioneiras ocorrem ao longo dos cursos d'água ou das depressões com água. No Estado essas áreas apresentam-se pouco significativas, registrando sua presença apenas nas ilhas do rio Paraná e nas áreas próximas aos rios Dourados e Brilhante.

As áreas de tensão ecológica têm distribuição generalizada, fato que se repete com relação às áreas antrópicas, representam contato entre duas ou mais regiões fitoecológicas e que se manifestam na forma de interpenetração de espécies (ecótono ou mistura) ou interpenetração, sem se misturar (encraves).

Geomorfologia e Relevo. O Estado apresenta quatro fisionomias distintas no relevo. A parte oriental compreende um relevo alçado constituído por planaltos, patamares e chapadões inseridos na Bacia Sedimentar do Paraná. De sua borda ocidental em direção oeste, estende-se vasta superfície rebaixada recoberta por sedimentos quaternários, a região do Pantanal sul-mato-grossense e a Depressão do Alto Paraguai. Em meio a essas regiões rebaixadas, erguem-se relevos elevados da Bodoquena e as Morrarias do Urucum-Amolar.

A origem e a evolução das diferentes fisionomias do relevo original acham-se intimamente relacionadas a movimentos de compensação isostática muito antigos, como o soerguimento dos Andes e da Bacia Sedimentar do Paraná e o abatimento entre os dois, onde se instalou a atual Bacia do Paraguai.

A hidrografia do Estado divide-se basicamente em duas 


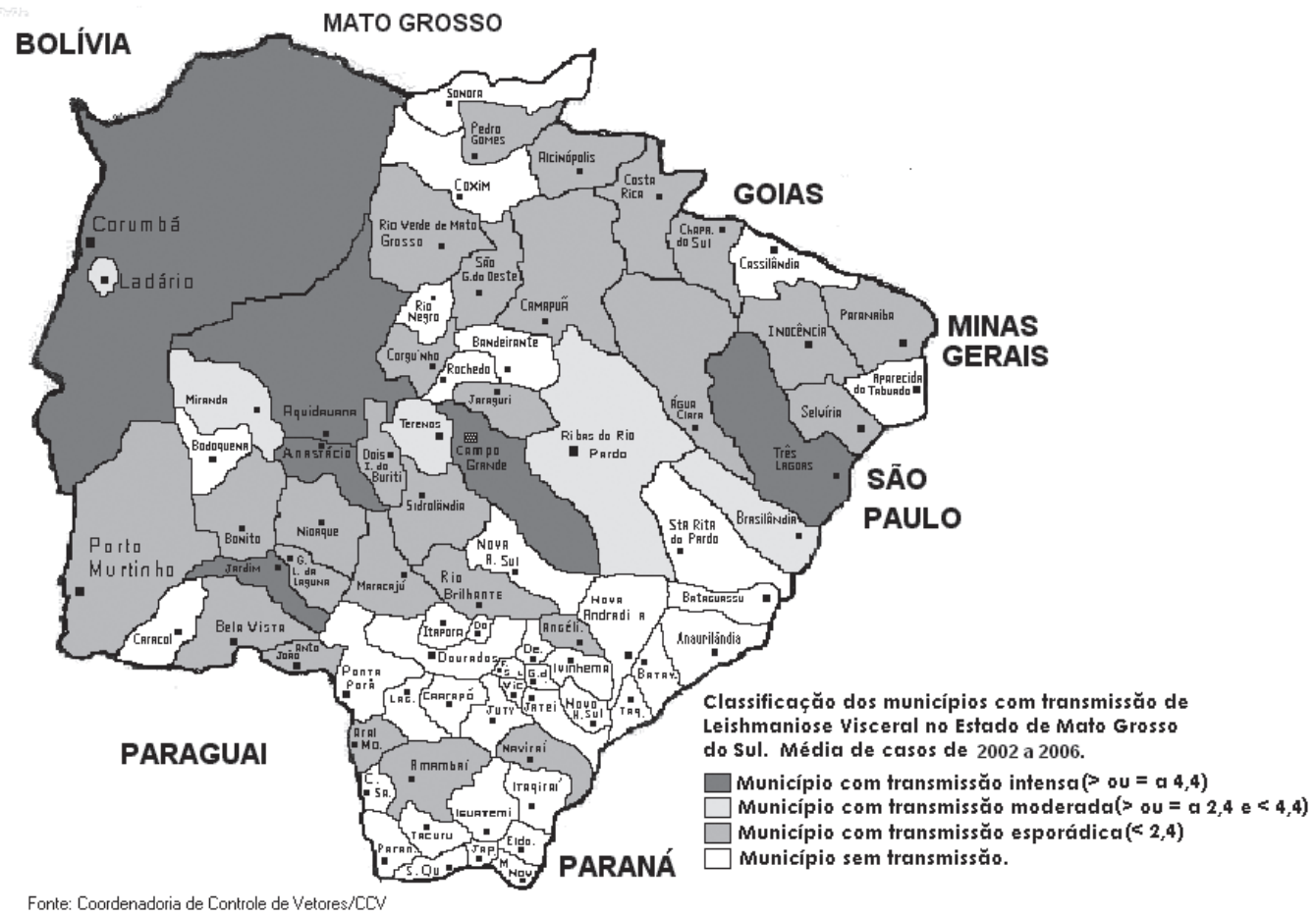

Fig. 1. Mapa do Estado de Mato Grosso do Sul, mostrando os municípios onde foram registrados casos de leishmaniose visceral, seguindo-se a classificação do Manual de vigilância e controle da leishmaniose visceral do Mistério da Saúde, no período de 2002 a 2006.

grandes bacias: do Paraná a leste e a do Paraguai a oeste. O divisor das águas compreende a Serra das Araras, a Serra de Camapuã e parte da Serra de Maracaju. Entre as duas bacias há um pronunciado desnível e o contato se dá através de escarpas.

\section{Municípios trabalhados}

Anastácio (20²9’ 01” S; 55 48’ 25” O; 160 m. a. n. m.) dista $128 \mathrm{~km}$ da capital Campo Grande, faz parte do pantanal sul-mato-grossense, possui uma área estimada em $2.949 \mathrm{~km}^{2}$, sua extensão representa $0,83 \%$ do Estado, sua população é de 24.352 habitantes (2006). O turismo, a pecuária e o comércio são as maiores fontes econômicas do município. Coletas realizadas no período de outubro de 2003 a setembro de 2005.

Aquidauana (20 28' $16^{\circ}$ ' S; $55^{\circ} 47^{\prime} 14^{\prime \prime} \mathrm{O} ; 147 \mathrm{~m}$. a. n. m.), dista $331 \mathrm{~km}$ da capital Campo Grande, faz parte do pantanal sul-mato-grossense, possui uma área estimada em $16.958 \mathrm{~km}^{2}$, sua extensão representa 4,75\% do Estado, sua população é de 46.469 habitantes (2006). Coletas realizadas no período de outubro de 2003 a setembro de 2005.

Bela Vista (22 06' 32" S; 56 31' 16” O; 180 m. a. n. m.), encontra-se a $349 \mathrm{~km}$ da capital Campo Grande; na mesorregião sudoeste do Estado, possui uma área estimada em $4.895 \mathrm{~km}^{2}$, sua extensão representa 1,37\% do Estado, sua população é de 23.707 habitantes (2006). Cidade turística faz fronteira com a República do Paraguai. Coletas realizadas no período de novembro de 2004 a outubro de 2006.

Bodoquena (20 33' 03” S; 56 40'06” O; $132 \mathrm{~m}$. a. n. m.), localiza-se a $253 \mathrm{~km}$ da capital Campo Grande, na mesorregião sudoeste do Estado, possui uma área estimada em $2.507 \mathrm{~km}^{2}$, sua extensão representa $0,70 \%$ do Estado, sua população em 2006, era de 8.550 habitantes. Coletas realizadas no período de outubro de 2003 a setembro de 2005 .
Bonito (21 07’ 16” S; 56 28' 55” O; 315 m. a. n. m.) situa-se a 297 km da capital Campo Grande, na mesorregião sudoeste do Estado, área de $4.934 \mathrm{~km}^{2}$, representa 1,38\% do Estado, sua população é de 18.000 habitantes (2006). Coletas realizadas no período de outubro de 2004 a setembro de 2006.

Brasilândia $\left(21^{\circ} 15^{\prime} 11^{\prime \prime} \mathrm{S} ; 52^{\circ} 02^{\prime} 13^{\prime \prime} \mathrm{O} ; 343 \mathrm{~m}\right.$. a. n. m.) dista $374 \mathrm{~km}$ da capital Campo Grande, na mesorregião leste do Estado, possui uma área estimada em $5.806 \mathrm{~km}^{2}$, sua extensão representa $1,63 \%$ do Estado, sua população em 2006 era de 13.144 habitantes. Coletas realizadas no período de outubro de 2003 a setembro de 2005.

Campo Grande (20²6’34” s; 54 38’ 47” O; 532 m. a. n. $\mathrm{m})$, capital do Estado, está situada na região centro-norte do Estado, possui uma área estimada em $8.096 \mathrm{~km}^{2}$, sua extensão representa 2,27\% do Estado, sua população em 2006 era de 765.247 habitantes. Coletas realizadas no período de outubro de 2003 a setembro de 2005.

Corumbá (1900’33” S; 57 39’ 12” O; 118 m. a. n. m) situa-se a $415 \mathrm{~km}$ da capital Campo Grande, na região do pantanal sul-mato-grossense, faz fronteira com a Bolívia e possui uma área estimada em $64.960 \mathrm{~km}^{2}$, sua extensão representa 18,19\% do Estado, sua população em 2006 era de 101.089 habitantes. Coletas realizadas no período de outubro de 2003 a setembro de 2005.

Guia Lopes da Laguna (21 27’ 28” S; 56 06’ 51” O; 272 m. a. n. m.) localiza-se a $233 \mathrm{~km}$ da capital Campo Grande, na mesorregião sudoeste do Estado, possui uma área estimada em $1.210 \mathrm{~km}^{2}$, sua extensão representa 0,34\% do Estado. Coletas realizadas no período de outubro de 2004 a setembro de 2006.

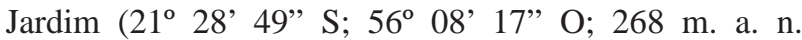
m.) encontra-se a $238 \mathrm{~km}$ da capital Campo Grande, na 
mesorregião sudoeste do Estado, possui uma área estimada em $2.201 \mathrm{~km}^{2}$, sua extensão representa $0,62 \%$ do Estado, sua população é de 24.920 habitantes (2006). Coletas realizadas no período de outubro de 2003 a setembro de 2005.

Ladário (1900’ 17” S; 57 36’ 06”; 114 m. a. n. m.) situase a $410 \mathrm{~km}$ da capital Campo Grande, na região do pantanal sul-mato-grossense, possui uma área estimada em $342 \mathrm{~km}^{2}$, sua extensão representa $0,10 \%$ do Estado, sua população é de 17.776 habitantes (2006). Coletas realizadas no período de outubro de 2003 a setembro de 2005.

Maracajú (21 36’ 52” S; 55 10’ 06” O; 384 m. a. n. m.) está distante $157 \mathrm{~km}$ da capital Campo Grande, na mesorregião sudoeste do Estado, possui uma área estimada em $5.298 \mathrm{~km}^{2}$, sua extensão representa 1,48\% do Estado, sua população é de 28.599 habitantes (2006).

Miranda (20 14' 26” s; 56 22' 42” O; 125 m. a. n. m.) localiza-se a $195 \mathrm{~km}$ da capital Campo Grande, na região do pantanal sul-mato-grossense, possui uma área estimada em $5.478 \mathrm{~km}^{2}$, sua extensão representa $1,53 \%$ do Estado, sua população é de 25.099 habitantes (2006). Coletas realizadas no período de novembro de 2004 a outubro de 2006.

Nioaque ( $21^{\circ} 08^{\prime} 07^{\prime \prime}$ S; 55 49’ 48” O; 200 m. a. n. m.) dista $177 \mathrm{~km}$ da capital Campo Grande, na mesorregião sudoeste do Estado, possui uma área estimada em 3.923 km², sua extensão representa $1,10 \%$ do Estado, sua população é de 18.064 habitantes (2006). Coletas realizadas no período de outubro de 2004 a setembro de 2006.

Ribas do Rio Pardo (20²9’01” S; 55 48’ 25” O; 160 m. a. n. m.) localiza-se a $84 \mathrm{~km}$ da capital Campo Grande, na mesorregião leste do Estado, possui uma área estimada em $17.308 \mathrm{~km}^{2}$, sua extensão representa 4,85\% do Estado, sua população é de 19.159 habitantes (2006). Coletas realizadas no período de outubro de 2003 a setembro de 2005.

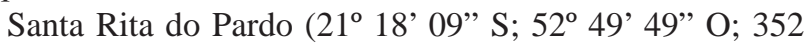
m. a. n. m.) está distante $258 \mathrm{~km}$ da capital Campo Grande, na mesorregião leste do Estado, possui uma área estimada em $6.141 \mathrm{~km}^{2}$, sua extensão representa 1,72\% do Estado, sua população é de 7.451 habitantes (2006). Coletas realizadas no período de outubro de 2003 a setembro de 2005.

Terenos (20 26' 32”' S; 54 51' 07” O; 372 m. a. n. m.) situa-se a $23 \mathrm{~km}$ da capital Campo Grande, na mesorregião centro-norte do Estado, possui uma área estimada em 2.841 $\mathrm{km}^{2}$, sua extensão representa $0,80 \%$ do Estado, sua população é de 12.892 habitantes (2006). Coletas realizadas no período de outubro de 2003 a setembro de 2005.

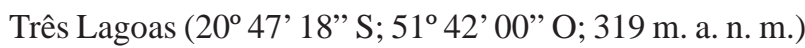
localiza-se a $313 \mathrm{~km}$ da capital Campo Grande, na mesorregião leste do Estado, considerado pelo Programa Nacional de Controle da Dengue município prioritário, possui uma área estimada em $10.206 \mathrm{~km}^{2}$, sua extensão representa 2,86\% do Estado, sua população é de 87.113 habitantes (2006). Coletas realizadas no período de outubro de 2003 a setembro de 2005.

Coleta e análise dos flebotomíneos - As coletas foram realizadas com armadilhas automáticas luminosas do tipo CDC, instalada mensalmente durante três noites consecutivas, das 18:00 às 6:00 horas, no período de dois anos, segundo preconizado pelo Ministério da Saúde (2003). A pesquisa foi realizada em pontos fixos da área urbana de 18 municípios do Estado com transmissão de leishmaniose visceral. A amostragem do número de pontos variou entre os municípios em função da área territorial urbana. Os municípios de Anastácio, Bela Vista, Bonito, Brasilândia, Bodoquena, Guia Lopes da Laguna, Ladário, Miranda, Maracajú, Nioaque, Ribas do Rio Pardo, Santa Rita do Parado e Terenos foram amostrados com quatro pontos fixos de coleta; Jardim com cinco pontos fixos; Aquidauana e Corumbá com seis pontos cada; Três Lagoas com oito pontos e Campo Grande com 12 pontos de coletas.

Os insetos foram identificados na Rede de Laboratórios Regionais de Entomologia da Secretaria Estadual de Saúde (SES-MS). A classificação utilizada seguiu a proposta por Galati (2003).

Foi feito o índice de diversidade de Simpson $\left(\mathrm{D}=1 / \sum \mathrm{p}_{\mathrm{i}}{ }^{2}\right)$ para cada município. $\mathrm{O}$ cálculo é feito a partir da frequência de cada espécie $\left(\mathrm{p}_{\mathrm{i}}\right)$ na amostra total de indivíduos e quando for próximo a 1 indica que a fauna é pouco diversa, com elevada proporção de uma espécie sobre as demais, sendo seu valor máximo igual ao número de espécies encontradas na amostra, o que indica que essas têm frequência igual na população estudada (Ricklefs 2003). Para se comparar se houve diferença significativa entre os sexos foi utilizado o teste não paramétrico de Mann-Whitney.

\section{RESULTADOS}

Foram capturados 34.799 espécimes de flebotomíneos distribuídas por 36 espécies (Tabela I), sendo 18 delas pertencentes à subtribo Lutzomyiina, 12 a Psychodopygina, três a Sergentomyiina e três à Brumptomyiina. Destas subtribos, a primeira contribuiu com a grande maioria dos flebotomíneos, num total de 34.183 (98,23\%) espécimes.

As espécies mais abundantes foram Lutzomyia longipalpis (Lutz \& Neiva, 1912) (56,33\%), seguida de Lutzomyia cruzi (Mangabeira, 1938) (37,95\%), Lutzomyia forattinii Galati, Rego, Nunes \& Teruya, 1985 (2,88\%) e Nyssomyia whitmani (Antunes \& Coutinho, 1939) (1,10\%), as demais espécies perfizeram $1,74 \%$.

Os machos foram mais numerosos (27.462) do que as fêmeas (7.337), sendo a razão macho: fêmea $=3,74$ : 1 . Aplicando-se o teste U de Mann-Whitney, os dados revelaram que houve um número médio de machos significativamente maior do que o de fêmeas $(Z=2,17 ; p=0,03)$.

Apesar do número diferente de municípios em cada mesorregião trabalhada, a Sudoeste e Pantanal apresentaram maior diversidade de espécies do que a Centro-norte e Leste. O mesmo é valido para as duas bacias hidrográficas analisadas, a do rio Paraguai apresentou maior variedade de espécies do que a do rio Paraná. Apesar disso, duas espécies, Evandromyia bacula (Martins, Falcão \& Silva, 1965) e Psathyromyia lutziana (Costa Lima, 1932) foram encontradas apenas na mesorregião Leste, conseqüentemente, apenas na bacia do rio Paraná (Tabela II).

O maior número de espécies foi encontrado em Aquidauana (16) e Bonito (15) enquanto em Ribas do Rio Pardo apenas duas espécies foram capturadas (Tabela I). $L u$. 
Tabela I. Flebotomíneos coletados em 18 municípios do Estado de Mato Grosso do Sul com armadilha luminosa CDC no período de dois anos.

\begin{tabular}{|c|c|c|c|c|c|c|c|c|c|c|c|c|c|c|c|c|c|c|c|}
\hline & & & & & & & & & unicípi & & & & & & & & & & Freqüência (\%) \\
\hline Espécies & 1 & 2 & 3 & 4 & 5 & 6 & 7 & 8 & 9 & 10 & 11 & 12 & 13 & 14 & 15 & 16 & 17 & 18 & \\
\hline$\overline{\text { Bi. flaviscutellata }}$ & & 2 & 1 & & 14 & & & & & & & & & & & 1 & & & 0,05 \\
\hline Br. avellari & & 2 & & & 1 & & 1 & & 1 & & & & & & 1 & 3 & & & 0,03 \\
\hline Br. brumpti & & & 2 & & 14 & 1 & 1 & & 13 & 1 & & 9 & & 5 & & 5 & & 3 & 0,16 \\
\hline Br. cunhai & & & & & 1 & & & & & & & & & & & & & & 0,00 \\
\hline Ev. aldafalcoae & 3 & 12 & & & & & & 1 & 43 & 8 & & & & 2 & & & & & 0,20 \\
\hline Ev. bacula & & & & & & & & & & & & & & & & 1 & & & 0,00 \\
\hline Ev. evandroi & & 2 & & 1 & & & & & & 1 & & & & & & & 1 & & 0,02 \\
\hline Ev. lenti & 3 & 2 & 1 & 1 & 6 & & 3 & & 19 & & & 2 & & 6 & & 1 & 1 & 2 & 0,14 \\
\hline Ev. termitophila & & & & & 1 & 1 & 1 & & & & & 1 & & 1 & & & & & 0,02 \\
\hline Ev. walkeri & & & & & & & & & & & & & & 3 & & & & 2 & 0,02 \\
\hline Ev. corumbaensis & & & & 4 & 16 & & & 39 & & & 3 & & 11 & 3 & & & & & 0,22 \\
\hline Ev. sallesi & 1 & 3 & & 1 & 31 & & & & 18 & & & 25 & 12 & 5 & & & & 10 & 0,30 \\
\hline Ev. cortelezzii & & 4 & & & & & & 3 & 11 & & & & & & & & & & 0,05 \\
\hline Lu. cruzi & & & & & 1 & & & 13164 & & & 41 & & & & & & & & 37,95 \\
\hline Lu. dispar & & 1 & & & & & & & & & & & & & & & & 3 & 0,01 \\
\hline Lu. forattinii & 1 & & & & & & & 1001 & & & 1 & & & & & & & & 2,88 \\
\hline Lu. longipalpis & 495 & 433 & 230 & 81 & 5034 & 435 & 2038 & & 1430 & 5414 & & 114 & 33 & 867 & 124 & 14 & 2074 & 787 & 56,32 \\
\hline Lu. almerioi & & & & & & & & 4 & & & & & & & & & & & 0,01 \\
\hline Mt. oliveirai & & & & & & & & 5 & & & & & & & & & & & 0,02 \\
\hline Mi. longipennis & & 1 & & & & & & & & & & & & & & & & & 0,00 \\
\hline Mi. quinquefer & & 1 & & & & & & & & & & & & & & & & & 0,00 \\
\hline Mi. peresi & & & & & & & 2 & 2 & & 1 & & 2 & & & & & & & 0,02 \\
\hline Mg. migonei & & & & 2 & & & & & & 1 & & & & & & & & & 0,01 \\
\hline Ny. whitmani & 3 & 10 & 1 & 79 & 36 & & 3 & & 10 & 100 & 4 & 5 & 11 & 11 & & 11 & 2 & 96 & 1,10 \\
\hline Ny. neivai & & 3 & & & 1 & 7 & & & & & & & & & & 5 & 1 & 1 & 0,05 \\
\hline Pi. pessoai & & 3 & & & & & & & & & & & & & & & & & 0,01 \\
\hline Pi. christenseni & & & & & & & & & & & & 1 & & & & & & & 0,00 \\
\hline Ps. aragaoi & & & & & 1 & & & 2 & & & & & & 1 & & & & & 0,01 \\
\hline Pa. hermanlenti & 1 & & & & & & & & & & & & & & & & & 3 & 0,01 \\
\hline Pa. lutziana & & & & & & & & & & & & & & & & & & 1 & 0,00 \\
\hline Pa. campograndensis & & & 2 & & & 2 & & & & 1 & 1 & & & & & 1 & & & 0,02 \\
\hline Pa. lanei & & & & & & & & & 1 & & & & & & & & & & 0,00 \\
\hline Pa. punctigeniculata & & & & & & & & & & 1 & & & & & & & & 1 & 0,01 \\
\hline Pa. scaffi & 1 & & & & & & & & & & & 15 & & & & & & & 0,05 \\
\hline Pa. shannoni & 7 & 4 & & 2 & 7 & & 39 & 11 & 2 & 10 & & 1 & & 2 & & & & & 0,24 \\
\hline Sc. sordellii & & 2 & & & 3 & & & & 5 & & & 6 & & 3 & & 2 & & 3 & 0,07 \\
\hline Freqüência (\%) & 1,48 & 1,39 & 0,68 & 0,49 & 14,85 & 1,28 & 6,00 & 40,92 & 4,46 & 15,91 & 0,14 & 0,52 & 0,19 & 2,61 & 0,36 & 0,13 & 5,97 & 2,62 & 100,00 \\
\hline Diversidade & 1,1 & 1,2 & 1,1 & 2,3 & 1,1 & 1,0 & 1,0 & 1,2 & 1,2 & 1,0 & 1,5 & 2,0 & 3,0 & 1,1 & 1,0 & 4,6 & 1,0 & 1,3 & \\
\hline
\end{tabular}

Legenda - 1 - Anastácio, 2 - Aquidauana, 3 - Bela Vista, 4 - Bodoquena, 5 - Bonito, 6 - Brasilândia, 7 - Campo Grande, 8 - Corumbá, 9 - Guia Lopes da Laguna, 10 - Jardim, 11 - Ladário, 12 - Maracajú, 13 - Miranda, 14 - Nioaque, 15 - Ribas do Rio Pardo, 16 - Santa Rita do Pardo, 17 - Três Lagoas, 18 - Terenos.

longipalpis foi encontrada em 16 municípios, sendo a espécie melhor distribuída pelo Estado, seguida de Ny whitmani (15), Evandromyia lenti (Mangabeira, 1938) (12), Brumptomyia brumpti (Larrousse, 1920) e Psathyromyia shannoni (Dyar, 1929), ambas encontrada em 10 municípios. Lutzomyia cruzi foi encontrada apenas em três municípios.

Corumbá foi o município que apresentou maior número de flebotomíneos capturados (14.232), distribuído por apenas três espécies, esse valor é sustentado pela grande quantidade de $L u$. cruzi (13.164) e Lu. forattinii (1.001) encontradas nesse município. Lutzomyia longipalpis esteve ausente em Corumbá e em Ladário e foi a espécie mais abundante em todas as demais localidade trabalhadas, sendo que em Santa Rita do Pardo e Bodoquena, Ny. whitmani foi coletada em frequência próxima a dessa espécies.

Os municípios que apresentaram maior índice de diversidade foram Santa Rita do Pardo $(D=4,63)$ e Miranda $(\mathrm{D}=3,04)$ e o menor em Três Lagoas $(\mathrm{D}=1,00)$ e Ribas do Rio Pardo $(\mathrm{D}=1,02)$ (Tabela I).

\section{DISCUSSÃO}

A fauna flebotomínica no Mato Grosso do Sul é relativamente bem conhecida e até o momento compõe-se de 54 espécies (Young \& Duncan 1994; Galati et al. 2006; Oliveira et al. 2006; Andrade Filho et al. 2007). Nesse trabalho registramos pela primeira vez a presença das espécies Ev. bacula, Evandromyia evandroi (Costa Lima \& Antunes, 1936), Evandromyia walkeri (Newstead, 1914), Migonemyia migonei (França, 1920) e Psathyromyia scaffi (Damasceno \& Arouck, 1956), aumentando para 59 o número de espécies de flebotomíneos encontradas para esse Estado. Com relação a essa última espécie, seu encontro foi inesperado, já que é uma espécie registrada apenas nos domínios amazônicos (Galati 2003), sendo aqui descrita para o cerrado do Estado de Mato Grosso do Sul, mesorregião Sudoeste.

Cada município pesquisado mostrou-se particular. Como exemplo, Três Lagoas, pertencente à mesorregião Leste, que apresentou maior diversidade de espécies quando comparado aos demais municípios dessa mesorregião, sendo ainda 
Tabela II. Distribuição das espécies de flebotomíneos coletadas por mesorregiões e bacia hidrográfica no Estado de Mato Grosso do Sul com armadilha luminosa CDC no período de dois anos.

\begin{tabular}{|c|c|c|c|c|c|c|}
\hline & \multicolumn{4}{|c|}{ Mesorregião } & \multicolumn{2}{|c|}{ Bacia hidrográfica } \\
\hline Espécie & $\begin{array}{c}\text { Centro- } \\
\text { Norte }\end{array}$ & Leste & Sudoeste & Pantanal & Paraná & Paraguai \\
\hline Bi. flaviscutellata & & $\mathrm{X}$ & $\mathrm{X}$ & $\mathrm{X}$ & $\mathrm{O}$ & $\mathrm{O}$ \\
\hline Br. avellari & $\mathrm{X}$ & $\mathrm{X}$ & $\mathrm{X}$ & $\mathrm{X}$ & $\mathrm{O}$ & $\mathrm{O}$ \\
\hline Br. brumpti & $\mathrm{X}$ & $\mathrm{X}$ & $\mathrm{X}$ & & $\mathrm{O}$ & $\mathrm{O}$ \\
\hline Br. cunhai & & & $\mathrm{X}$ & & & $\mathrm{O}$ \\
\hline Ev. aldafalcoae & & & $\mathrm{X}$ & $\mathrm{X}$ & & $\mathrm{O}$ \\
\hline Ev. bacula & & $\mathrm{X}$ & & & $\mathrm{O}$ & \\
\hline Ev. evandroi & $\mathrm{X}$ & & $\mathrm{X}$ & $\mathrm{X}$ & & $\mathrm{O}$ \\
\hline Ev. lenti & $\mathrm{X}$ & $\mathrm{X}$ & $\mathrm{X}$ & $\mathrm{X}$ & $\mathrm{O}$ & $\mathrm{O}$ \\
\hline Ev. termitophila & $\mathrm{X}$ & $\mathrm{X}$ & $\mathrm{X}$ & & $\mathrm{O}$ & $\mathrm{O}$ \\
\hline Ev. walkeri & & $\mathrm{X}$ & $\mathrm{X}$ & & & $\mathrm{O}$ \\
\hline Ev. corumbaensis & & & $\mathrm{X}$ & $\mathrm{X}$ & & $\mathrm{O}$ \\
\hline Ev. sallesi & & $X$ & $\mathrm{X}$ & $\mathrm{X}$ & $\mathrm{O}$ & $\mathrm{O}$ \\
\hline Ev. cortelezzii & & & $\mathrm{X}$ & $\mathrm{X}$ & & $\mathrm{O}$ \\
\hline Lu. cruzi & & & $\mathrm{X}$ & $\mathrm{X}$ & & $\mathrm{O}$ \\
\hline Lu. dispar & & $\mathrm{X}$ & & $\mathrm{X}$ & $\mathrm{O}$ & $\mathrm{O}$ \\
\hline Lu. forattinii & & & & $\mathrm{X}$ & & $\mathrm{O}$ \\
\hline Lu. longipalpis & $\mathrm{X}$ & $\mathrm{X}$ & $\mathrm{X}$ & $\mathrm{X}$ & $\mathrm{O}$ & $\mathrm{O}$ \\
\hline Lu. almerioi & & & & $\mathrm{X}$ & & $\mathrm{O}$ \\
\hline Mt. oliveirai & & & & $X$ & & $\mathrm{O}$ \\
\hline Mi. longipennis & & & & $\mathrm{X}$ & & $\mathrm{O}$ \\
\hline Mi. quinquefer & & & & $\mathrm{X}$ & & $\mathrm{O}$ \\
\hline Mi. peresi & $\mathrm{X}$ & & $\mathrm{X}$ & $\mathrm{X}$ & $\mathrm{O}$ & $\mathrm{O}$ \\
\hline Mg. migonei & & & $\mathrm{X}$ & & & $\mathrm{O}$ \\
\hline Ny. whitmani & $\mathrm{X}$ & $\mathrm{X}$ & $\mathrm{X}$ & $\mathrm{X}$ & $\mathrm{O}$ & $\mathrm{O}$ \\
\hline Ny. neivai & $\mathrm{X}$ & $X$ & $\mathrm{X}$ & $\mathrm{X}$ & $\mathrm{O}$ & $\mathrm{O}$ \\
\hline Pi. pessoai & & & & $X$ & & $\mathrm{O}$ \\
\hline Pi. christenseni & & & $\mathrm{X}$ & & & $\mathrm{O}$ \\
\hline Ps. aragaoi & & & $\mathrm{X}$ & $\mathrm{X}$ & & $\mathrm{O}$ \\
\hline Pa. hermanlenti & & $\mathrm{X}$ & & $\mathrm{X}$ & $\mathrm{O}$ & $\mathrm{O}$ \\
\hline Pa. lutziana & & $\mathrm{X}$ & & & $\mathrm{O}$ & \\
\hline Pa. campograndensis & & $\mathrm{X}$ & $\mathrm{X}$ & $\mathrm{X}$ & $\mathrm{O}$ & $\mathrm{O}$ \\
\hline Pa. lanei & & & $\mathrm{X}$ & & & $\mathrm{O}$ \\
\hline Pa. punctigeniculata & & $\mathrm{X}$ & $\mathrm{X}$ & & $\mathrm{O}$ & $\mathrm{O}$ \\
\hline Pa. scaffi & & & $\mathrm{X}$ & $\mathrm{X}$ & & $\mathrm{O}$ \\
\hline Pa. shannoni & $\mathrm{X}$ & & $\mathrm{X}$ & $\mathrm{X}$ & $\mathrm{O}$ & $\mathrm{O}$ \\
\hline Sc. sordellii & & $\mathrm{X}$ & $\mathrm{X}$ & $\mathrm{X}$ & $\mathrm{O}$ & $\mathrm{O}$ \\
\hline Total de espécies & 10 & 17 & 26 & 26 & 18 & 34 \\
\hline
\end{tabular}

que duas espécies foram encontradas apenas nesse local. A grande variabilidade específica encontrada em todo Estado certamente é reflexo da alta heterogeneidade espacial, com a presença de vários biomas e ecótopos. Isso demonstra a necessidade de se realizar trabalhos específicos sobre a fauna de flebotomíneos e seus aspectos ecológicos em cada município de interesse, não só nesse Estado, mas em todo Brasil, tentando-se dessa maneira compreender melhor a complexa relação parasita/vetor/hospedeiro.

Galati et al. (2003a, 2006) trabalharam na Serra da Bodoquena, registrando 25 espécies com predomínio absoluto de Lutzomyia almerioi Galati \& Nunes, 1999 em área de caverna. Essa espécie foi encontrada naturalmente infectada pela Leishmania chagasi e Leishmania (Viannia) sp. (Savani et al. 2005) e, nessa mesma localidade, em áreas florestais, além desta espécie, foi capturada $N y$. whitmani, Lu. longipalpis e Evandromyia corumbaensis (Galati, Nunes, Oshiro \& Rego, 1989).

Para os ecossistemas Pantanal já foram encontradas 16 espécies, com predomínio na área urbana de Corumbá das espécies $L u$. cruzi, Lu. forattinii e Ev. corumbaensis (Galati et al. 1997; Santos et al. 1998) e em área rural deste município de Brumptomyia avellari (Costa Lima, 1932), Ev. lenti e Evandromyia aldafalcaoae (Santos, Andrade Filho \& Honer, 2001) (Braga-Miranda et al. 2006). Fato interessante é o encontro em Corumbá de Evandromyia cortelezzii (Brèthes, 1923), espécie que forma o complexo cortelezzii juntamente com Evandromyia sallesi (Galvão \& Coutinho, 1939) e Evandromyia corumbaensis (Galati, Nunes, Oshiro \& Rego, 1989) (Galati et al. 1989). Apenas as duas últimas espécies citadas haviam sido registradas nesse município (Galati et al. 1997, 2003a), assim como Lu. almerioi, normalmente encontrada na Serra da Bodoquena e arredores (Galati et al. 2003b, 2006).

A ocorrência de uma espécie numa comunidade local é determinada parcialmente por suas adaptações às condições e recursos e parcialmente por interações competitivas com outras espécies (Ricklefs 2003). Esse processo de seleção de espécies pode explicar a ausência de Lu. longipalpis em dois municípios trabalhados, onde foi encontrada a espécie $\mathrm{Lu}$. cruzi. Por serem muito próximas filogeneticamente, ambas devem utilizar os mesmos recursos ecológicos, e por competição, ocorre a eliminação de uma delas. Além de predominar em dois municípios na ausência de $L u$. longipalpis, $L u$. cruzi só foi encontrada em um terceiro município e em uma freqüência muita baixa.

Os dados apresentados aqui para $\mathrm{Lu}$. longipalpis em Campo Grande são basicamente os mesmos utilizados por Silva et al. (2007). É interessante notar que o padrão apresentado para $L u$. longipalpis, com grande prevalência sobre as demais espécies, é diferente daquele registrado por Oliveira et al. (2000; 2003), onde ela não era a espécie mais freqüente, o que vem a mostrar a grande adaptação dessa espécie ao ambiente urbano, o que já havia sido demonstrado por Oliveira et al. (2006). Ainda nesse município $L u$. longipalpis foi mais encontrada após o período de fortes chuvas (dado não apresentado), quando é recomendado que sejam aplicadas as medidas de controle contra o vetor (Oliveira et al. 2008).

É importante destacar ainda que dentre as espécies mais freqüentes encontradas nesse estudo estão as principais vetoras (ou suspeitas de o serem) de espécies de Leishmania no Brasil. Lutzomyia longipalpis e Lu. cruzi estão envolvidas na cadeia epidemiológica da LV no Brasil (Deane \& Deane 1954; Santos et al. 1998), assim como $L u$. forattinii e $L u$. almerioi espécies que já foram encontradas naturalmente infectadas com Leishmania infantum chagasi (Pita-Pereira et al. 2008; Savani et al. 2005). Nyssomyia whitmani está envolvida na veiculação de LTA em várias regiões do Brasil (Luz et al. 2000; Carvalho et al. 2008), o mesmo pode ser dito com relação a Mi. migonei, Nyssomyia neivai (Pinto, 1926) e $P a$. shannoni que foram encontradas em menor número, mas não devem ser ignoradas, pois apresentam potencial epidemiológico (Arias et al. 1985; Andrade Filho et. al. 2007; Pita-Pereira et al. 2005). As espécies do complexo cortelezzii, recentemente encontradas naturalmente infectadas (Carvalho et al. 2008; Saraiva et al. 2009), também merecem mais investigações.

Esse trabalho foi realizado em ambiente urbano, próximos às residências, isso justifica o encontro em freqüência baixa 
de algumas espécies e mostra, mais uma vez, a grande adaptabilidade e importância médica de Lu. longipalpis e $L u$. cruzi, comprovada pelo aumento do número de casos de LV em Mato Grosso do Sul.

\section{REFERÊNCIAS}

Andrade Filho, J. D.; E. A. B. Galati \& A. L. Falcão. 2007. Nyssomyia intermedia (Lutz \& Neiva, 1912) and Nyssomyia neivai (Pinto, 1926) (Diptera: Psychodidae: Phlebotominae) geographical distribution and epidemiological importance. Memórias do Instituto Oswaldo Cruz 102: 481-487.

Arias, J. R.; M. A. Miles; R. D. Naiff; M. M. Povoa; R. A. Freitas; C. B. Biancardi \& E. G. Castellon. 1985. Flagellate Infections of Brazilian Sand Flies (Diptera: Psychodidae): Isolation in vitro and biochemical identification of Endotrypanum and Leishmania. American Journal of Tropical Medicine and Hygine 34: 1098-1108.

Bagnouls, F. \& H. Gaussen. 1957. Les climats ecologiques et leur classification. Annales de Géographie 66: 193-220.

Braga-Miranda, L. C.; M. Miranda \& E. A. B. Galati. 2006. Phlebotomine fauna in a rural area of the Brazilian Pantanal. Revista Saúde Pública 40: 324-326.

Carvalho, G. M. L.; J. D. Andrade Filho; A. L. Falcão; A. C. V. M. Lima \& C. M. F. Gontijo. 2008. Naturally infected Lutzomyia sand flies in a Leishmania-endemic area of Brazil. Vector Borne and Zoonotic Diseases 8: 407-414.

Deane M. P. \& L. M. Deane 1954. Infecção natural do Phlebotomus longipalpis por lectomonas, provavelmente de Leishmania donovani, em foco de calazar, no Ceará. Hospital 45: 697-702.

Dorval, M. E. M. C.; E. T. Oshiro; E. Cupollilo; A. C. C. Castro \& T. P. Alves. 2006. Ocorrência de leishmaniose tegumentar americana no Estado de Mato Grosso do Sul associada à infecção por Leishmania (Leishmania) amazonensis. Revista da Sociedade Brasileira de Medicina Tropical 39: 43-46.

Dorval, M. E. C.; T. P. Alves; A. G. Oliveira; R. P. Brazil; E. A. B. Galati \& R. V. Cunha. 2007. Modification of Disney trap for capture of sand flies (Diptera: Psychodidae: Phlebotominae). Memórias do Instituto Oswaldo Cruz 102: 877-878.

Galati, E. A. B. 2003. Morfologia e Taxonomia. Classificação de Phlebotominae, p. 23-51. In: Rangel, E. F. \& R. Laionson (eds.). Flebotomíneos do Brasil. Rio de Janeiro, Editora Fiocruz, 367 p.

Galati, E. A. B.; V. L. B. Nunes; E. T. Oshiro; F. A. Rego Jr. 1989. Novas espécies de Phlebotominae, Lutzomyia corumbaensis sp. n. (Diptera, Psychodidae) do complexo cortelezzii. Revista Brasileira de Entomologia 33: 465-475.

Galati, E. A. B.; V. L. B. Nunes; F. A. Rego Jr; E. T. Oshiro \& M. R. Chang. 1997. Estudo de flebotomíneos (Diptera: Psychodidae) em foco de leishmaniose visceral no Estado de Mato Grosso do Sul. Revista de Saúde Pública 31: 378-390.

Galati E. A. B.; V. L. B. Nunes; C. Cristaldo \& H. C. Rocha. 2003a. Aspectos do comportamento da fauna flebotomínea (Diptera: Psychodidae) em foco de leishmaniose visceral e tegumentar na Serra da Bodoquena e área adjacente, Estado de Mato Grosso do Sul, Brasil. Revista de Patologia Tropical 32: 235-261.

Galati, E. A. B.; V. L. B. Nunes; P. C. Boggiani; M. E. C. Dorval; G. Cristaldo; H. C. Rocha; E. T. Oshiro; R. M. Gonçalves de Andrade \& G. Naufel. 2003b. Phlebotomines (Diptera, Psychodidae) in caves of the Serra da Bodoquena, Mato Grosso do Sul State, Brazil. Revista Brasileira de Entomologia 47: 283-296

Galati E. A. B.; V. L. B. Nunes; P. C. Bogiani; M. E. M. C. Dorval; G. Cristaldo; H. C. Rocha; E. T. Oshiro \& G. A. Damasceno-Junior. 2006. Phlebotomines (Diptera: Psychodidae) in forested areas of the Serra da Bodoquena, state of Mato Grosso do Sul, Brazil. Memórias do Instituto Oswaldo Cruz 101: 175-193.
Governo do Estado do Mato Grosso do Sul. 2007. Disponível em: <http:// www.ms.gov.br>. Acessado em 12/05/2010.

IBGE - Instituto Brasileiro de Geografia e Estatística. 2007. Disponível em: $<$ http://www.ibge.gov.br $>$. Acessado em 15/06/2007.

Luz, E.; N. Membrive; E. A. Castro; J. Dereure; F. Pratlong; A. Dedet; A. Pandey \& V. Thomaz-Soccol. 2000. Lutzomyia whitmani (Diptera: Psychodidae) as vector of Leishmania (V.) braziliensis in Paraná state, southern Brazil. Annals of Tropical Medicine and Parasitology 94: 623-631.

Ministério da Saúde. 2003. Manual de vigilância e controle da leishmaniose visceral. Brasília, $120 \mathrm{p}$.

Oliveira, A. G.; A. L. Falcão \& R. P. Brazil. 2000. Primeiro encontro de Lutzomyia longipalpis (Lutz \& Neiva, 1912) na área urbana de Campo Grande, MS, Brasil. Revista de Saúde Pública 34: 654-655.

Oliveira A. G.; J. D. Andrade Filho; A. L. Falcão \& R. P. Brazil. 2003. Estudos de flebotomíneos (Diptera, Psychodidae, Phlebotominae) na zona urbana de cidade de Campo Grande, Mato Grosso do Sul, 19992000. Cadernos de Saúde Pública 19: 933-944.

Oliveira,A. G.; E. A. B. Galati; O. Oliveira; G. R. Oliveira; I. A. C.Espíndola; M. E. C. Dorval \& R. P. Brazil. 2006. Abundance of Lutzomyia longipalpis (Diptera: Psychodidae: Phlebotominae) and urban transmission of visceral leishmaniasis in Campo Grande, state of Mato Grosso do Sul, Brazil. Memórias do Instituto Oswaldo Cruz 101: 869-874.

Oliveira, A. G.; E. A. B. Galati; C. E. Fernandes; M. E. C. Dorval \& R. P. Brazil. 2008. Seasonal variation of Lutzomyia longipalpis (Lutz \& Neiva, 1912) (Diptera: Psychodidae: Phlebotominae) in endemic area of visceral leishmaniasis, Campo Grande, state of Mato Grosso do Sul, Brazil. Acta Tropica 105: 55-61.

Pita-Pereira, D.; C. R. Alves; M. B. Souza; R. P. Brazil; A. L. Bertho; A. F. Barbosa \& C. C. Britto 2005. Identification of naturally infected Lutzomyia intermedia and Lutzomyia migonei with Leishmania (Viannia) braziliensis in Rio de Janeiro (Brazil) revealed by a PCR multiplex non-isotopic hybridisation assay. Transactions of the Royal Society of Tropical Medicine and Hygiene 99: 905-913.

Pita-Pereira, D.; M. A. Cardoso; C. R. Alves; R. P. Brazil \& C. C. Britto. 2008 Detection of natural infection in Lutzomyia cruzi and Lutzomyia forattinii (Diptera: Psychodidae: Phlebotominae) by Leishmania infantum chagasi in an endemic area of visceral leishmaniasis in Brazil using a PCR multiplex assay. Acta Tropica 107: 66-69.

Ricklefs, R. E. 2003. A economia da natureza. Rio de Janeiro, Editora Guanabara Koogan, 503 p.

Santos S. O.; J. Arias; A. Ribeiro; M. P. Hoffmann; R. A. Freitas \& M. A. F. Malacco. 1998. Incrimination of Lutzomya cruzi as a vector of American visceral leishmaniasis. Medical and Veterinary Entomology 12: 315317.

Saraiva, L.; G. M. L. Carvalho; C. M. F. Gontijo; P. F. Quaresma; A. C. V. M. R. Lima; A. L. Falcão \& J. D. Andrade Filho. 2009. Natural infection of Nyssomyia neivai and Evandromyia sallesi (Diptera: Psychodidae) by Leishmania infantum chagasi in Brazil. Journal of Medical Entomology 46: 1159-1163.

Savani, E. S. M. M.; E. A. B. Galati; V. L. B. Nunes; T. M. Castilho; M. C. O. Camargo; S. R. N. D’Auria \& L. M. Floeter-Winter. 2005. Natural infection in sand fly vectors of cutaneous and visceral leishmaniasis foci in Mato Grosso do Sul state, Brazil. Infecção natural de flebotomíneos em foco de leishmaniose cutânea e visceral no estado de Mato Grosso do Sul, Brasil. Proceedings of the V International Symposium on Phlebotomine sandflies. Archives de l' Institute Pasteur de Tunis 82 : 48-49.

Silva, E. A.; R. Andreotti \& M. R. Honer. 2007. Comportamento de Lutzomyia longipalpis, vetor principal da leishmaniose visceral americana, em Campo Grande, Estado do Mato Grosso do Sul. Revista da Sociedade Brasileira de Medicina Tropical 40: 420-425.

Young, D. G. \& Ducan, M. A. 1994. Guide to the Identification and Geographic Distribucion of Lutzomyia Sand Files in México, the West Indies, Central and South America (Diptera: Psychodidae). Florida, Memoirs of the American Entomological Institute. Number 54, $881 \mathrm{p}$.

Recebido em 09/09/2008; aceito em 15/04/2010

Editor: Mário Antonio Navarro da Silva 